Check for updates

Cite this: RSC Adv., 2019, 9, 4530

Received 12th November 2018

Accepted 24th January 2019

DOI: $10.1039 / c 8 r a 09329 f$

rsc.li/rsc-advances

\section{Insights into interaction of chlorophylls with sodium caseinate in aqueous nanometre-scale dispersion: color stability, spectroscopic, electrostatic, and morphological properties}

\begin{abstract}
Siyu He, (D) Nan Zhang and Pu Jing*
Chlorophylls are the major pigments present in photosynthetic plants but their application in foods is limited due to their lack of solubility in aqueous media and their susceptibility to degradation during processing and storage. These problems might be overcome by the addition of sodium caseinate (NaCas) whose hydrophobic and hydrophilic groups may result in electrostatic and steric stabilization. In the present work, $1 \%$ and $3 \%(\mathrm{w} / \mathrm{w})$ chlorophylls in $\mathrm{NaCas}$ dispersion or in ethanol (control) were prepared and their color stability under light treatment for $5 \mathrm{~h}$ and light-shed storage for 10 days was studied along with their interaction mechanism, using electrostatic, spectroscopic and morphological methodologies. Chlorophylls remained $58.72 \%$ and $53.84 \%$ in NaCas dispersions compared with the control $41.29 \%$ and 45.93\%) after light treatment for low- and high-dose treatment, respectively, suggesting that $\mathrm{NaCas}$ improved the solubility and stability of the pigments. Additionally, $1 \%$ and $3 \%(\mathrm{w} / \mathrm{w})$ chlorophylls in $\mathrm{NaCas}$ dispersion remained stable at $60.49 \%$ and $57.62 \%$ compared with the control $(44.81 \%$ and $48.17 \%)$ for the 10 day storage during which the zeta-potential of the dispersions changed from -31.7 to $-52.2 \mathrm{mV}$ and from -36.7 to $-56.2 \mathrm{mV}$ with a well-defined diameter ( 221-245 nm). The data obtained from electron microscopy, together with the results of fluorescence spectroscopy, suggests that chlorophylls were entrapped in $\mathrm{NaCas}$ dispersions mainly via hydrogen bonds.
\end{abstract}

\section{Introduction}

Chlorophylls are a class of naturally occurring pigments existing in all photosynthetic plants, and also in some bacteria and algae. They are present in greater abundance than any other organic pigments produced in nature. ${ }^{1,2}$ Chlorophylls can be used as potential alternatives to green colorants due to their naturally brilliant green color. However, when chlorophylls are extracted from plants they are highly susceptible to degradation due to heat, light, oxygen, acid, and enzymes. ${ }^{3,4}$ Moreover, chlorophylls are known to possess a wide range of pharmacological properties, such as anti-oxidant, anti-bacterial, antiinflammatory, anti-proliferative activities, deodorizing, and wound healing activities. ${ }^{5-7}$ Despite all these health benefits, there are limitations to the commercial-scale application of chlorophylls as natural colorants because they are highly susceptible to degradation during storage and processing, resulting in color changes in food. ${ }^{7}$ Although sodium copper chlorophyllin has been used, as a food colorant, in China and

Research Center for Food Safety and Nutrition, Key Lab of Urban Agriculture (South), Bor S. Luh Food Safety Research Center, School of Agriculture \& Biology, Shanghai Jiao Tong University, Shanghai 200240, China. E-mail: pjing@sjtu.edu.cn; Tel: +86 21 34207074
Europe as an excellent commercial alternative to chlorophylls because of its better solubility and stability, it is not approved in the US due to safety issues. ${ }^{8,9}$ Therefore, a safer form of soluble chlorophylls is currently required for the food industry. Several techniques have been reported to increase the aqueous solubility and stability of chlorophylls. Dissolving chlorophylls in lipids could decrease their light degradation since lipids compete for single oxygen with chlorophylls. ${ }^{10}$ Interacting chlorophylls with water-soluble proteins can improve the water solubility of chlorophylls as previously reported. ${ }^{11}$ Encapsulating chlorophylls in different proportions of gum arabic and maltodextrin by spray-drying has been shown to improve the storage stability of chlorophylls. ${ }^{7}$ Despite these great developments, much work is still needed in the preparation of dispersions to improve the aqueous solubility and stability of chlorophylls for food applications. Chlorophylls could be encapsulated by naturally occurring food biopolymers, which are generally recognized as safe ingredients.

Sodium caseinate (NaCas), a commercially available caseinrich ingredient, has been reported as a food-grade stabilizer in food and beverage industries. ${ }^{12}$ It is a naturally occurring amphiphilic block copolymer commonly utilized to stabilize hydrophobic bioactive compounds. ${ }^{13,14}$ This property makes it a good stabilizer with its hydrophobic and hydrophilic groups 
for contact with hydrophobic small molecules in aqueous systems. ${ }^{14,15}$

The current work was focused on evaluating the color stability, electrostatic, spectroscopic, and morphological properties of NaCas-chlorophylls dispersions using ultravioletvisible spectroscopy (UV-vis), transmission electron microscopy (TEM), scanning electron microscopy (SEM), atomic force microscopy (AFM), fluorescence spectroscopy, and Zeta Sizer. Based on the results obtained, the schematic mechanism of interaction between NaCas and chlorophylls was also proposed.

\section{Experimental section}

\subsection{Chemicals and reagents}

NaCas was purchased from Shanghai Titan Polytron Technologies Inc (RG). Chlorophylls $(0.5 \%, \mathrm{w} / \mathrm{w})$ were purchased from Sam Chemical Technology (Shanghai) Co., Ltd. Ethanol (purity $>99.8 \%$ ) was purchased from Sigma-Aldrich. All the other chemicals used were of analytical grade. All of the solutions were prepared with deionized water.

\subsection{Preparation of NaCas-chlorophylls dispersions}

The anti-solvent technique was used to fabricate the NaCaschlorophylls dispersion. ${ }^{16}$ Briefly, $0.1 \mathrm{~g}$ and $0.3 \mathrm{~g}$ of chlorophylls were dissolved in $3.5 \mathrm{~mL}$ of ethanol, followed by ultrasonic vibration ( $200 \mathrm{w}, 5 \mathrm{~min}$ ) and filtration through a $0.22 \mu \mathrm{m}$ Millipore microporous filter to obtain alcoholic solutions of the chlorophylls. $0.2 \mathrm{~g}$ of NaCas was dissolved in $6.5 \mathrm{~mL}$ of deionized water. The NaCas solution was stirred using a magnetic stirrer at room temperature $\left(\sim 25{ }^{\circ} \mathrm{C}\right)$ for $\sim 12 \mathrm{~h}$ and then centrifuged at $13010 \mathrm{~g}$ for $15 \mathrm{~min}$ at $4{ }^{\circ} \mathrm{C}$ to obtain a transparent supernatant. $3.5 \mathrm{~mL}$ of chlorophylls alcoholic solution was added to $6.5 \mathrm{~mL}$ of NaCas solution and the solution was mixed well under continuous magnetic stirring (100 $\left.\mathrm{r} \mathrm{min}^{-1}\right)$ for $10 \mathrm{~min}$. The final concentration of the chlorophylls in the mixture was $0 \%, 1 \%$, and $3 \%(\mathrm{w} / \mathrm{w})$ with $2 \%(\mathrm{w} / \mathrm{w})$ NaCas. Additionally, $3.5 \mathrm{~mL}$ of the chlorophylls alcoholic solution was mixed with $6.5 \mathrm{~mL}$ of deionized water control under the same conditions and the final chlorophylls concentration was $1 \%$ and $3 \%(\mathrm{w} / \mathrm{w})$. All the samples and controls were freeze-dried using a vacuum freeze dryer (Ningbo Xinzhi Biological Polytron Technologies, Inc.) to remove the solvent, and then dispersed in water to visually observe the dispersibility.

\subsection{Storage stability of NaCas-chlorophylls dispersions}

Freeze-dried samples were dissolved in deionized water in $5 \mathrm{~mL}$ clear glass vials and kept in a sterile operation room to reduce microbial spoilage. The particle size and zeta-potential of the dispersions were tested using a Zeta Sizer Nano (Malvern Instrument, ZS 90, UK) every day during the storage $\left(30^{\circ} \mathrm{C}\right)$ for ten days. Parameter settings: protein concentration, $5 \mathrm{mg} \mathrm{mL}^{-1}$; light source, $4 \mathrm{~mW} \mathrm{He}-\mathrm{Ne}$ laser; scattering angle, $90^{\circ}$; sample cell, $10 \mathrm{~mm}$ path length quartz cuvette and temperature, $25^{\circ} \mathrm{C}^{.17}$

\subsection{Photostability testing}

The effect of light on the physicochemical stability of the dispersions and chlorophylls was studied using a fluorescent lamp (9000 lx) for $0,0.5,1,2,4$, and $5 \mathrm{~h}$ at $25{ }^{\circ} \mathrm{C}$ in a BSG-250 incubator (BoXun, China). The light was a much higher intensity than normal sunlight. Freeze-dried samples were dissolved in transparent glass vials. The vials were placed at a distance of $40 \mathrm{~cm}$ away from the lamp. After exposure to light, the samples were kept in the dark at $25{ }^{\circ} \mathrm{C}$ for further testing.

\subsection{UV spectroscopy}

An L5S UV-vis spectrophotometer (Yidian, Shanghai, China) was used to measure the absorbance of the chlorophylls. The samples, after light or storage treatment, were freeze-dried and then dissolved in $2 \mathrm{~mL}$ of ethanol. All the solutions were kept in the dark for $2 \mathrm{~h}$ and ultrasonically treated using an XO-SM200 instrument (Nanjing Xian'ou, China) at $1200 \mathrm{~W}$ for $15 \mathrm{~min}$ at $25{ }^{\circ} \mathrm{C}$ under condensate circulation. The absorbance values of the solutions were measured at wavelengths of 646 and $662 \mathrm{~nm}$ at room temperature $\left(25{ }^{\circ} \mathrm{C}\right)$. Chlorophyll a and b were the major components of the chlorophylls and were found to be present at an approximate ratio of $3: 1$, the content of which was calculated using the following formulae: ${ }^{7,18}$

$$
\begin{aligned}
& \text { Chlorophyll a }\left(\mathrm{g} \mathrm{L}^{-1}\right)=11.24 \times A_{662}-2.04 \times A_{646} \\
& \text { Chlorophyll b }\left(\mathrm{g} \mathrm{L}^{-1}\right)=20.13 \times A_{646}-4.19 \times A_{662} \\
& \text { Retention rate }(\%)=(\mathrm{chl} \mathrm{a}+\mathrm{chl} \mathrm{b})_{\mathrm{T}} /(\mathrm{chl} \mathrm{a}+\mathrm{chl} \mathrm{b})_{0}
\end{aligned}
$$

where $A_{662} / A_{646}$ is the absorbance value at $662 / 646 \mathrm{~nm}$ (chl a + chl b) $)_{\mathrm{T}}$ is the amount of the chlorophylls after treatment and $(\mathrm{chl} \mathrm{a}+\mathrm{chl} \mathrm{b})_{0}$ is the initial amount of the chlorophylls.

\subsection{Fluorescence spectroscopy}

The intrinsic fluorescence of the chlorophylls in the dispersions was evaluated using a spectrophotometer (SpectraMax M2e, Sunnyvale, CA, USA) at various temperatures 25,35 , and $45^{\circ} \mathrm{C}$, as previously reported. ${ }^{19}$ The NaCas-chlorophylls samples were diluted with deionized water to an overall solute concentration of $0.2 \%(\mathrm{w} / \mathrm{w})$ to reach the range of instrument sensitivity. The samples were illuminated at an excitation wavelength of $280 \mathrm{~nm}$ and the resulting emission spectra were recorded from 290 to $450 \mathrm{~nm}$ using $5 \mathrm{~nm}$ slit widths. ${ }^{20}$ All the samples were evaluated in triplicate.

\subsection{Atomic force microscopy (AFM)}

The surface morphology of NaCas with or without chlorophylls was tested using an AFM (Multimode NanoscopeIIIa, Bruker). Briefly, NaCas, NaCas with $1 \%$ (w/w) chlorophylls (L-NaCas) and NaCas with $3 \%(\mathrm{w} / \mathrm{w})$ chlorophylls (H-NaCas) were diluted to solute concentration of $0.002 \%(\mathrm{w} / \mathrm{w})$ NaCas. Ten microliters of the diluted samples were spread evenly on a $1.8 \mathrm{~cm}^{2}$ freshlycleaved mica sheet and dried in a fume hood at ambient temperature $\left(25^{\circ} \mathrm{C}\right)$ overnight. ${ }^{21}$ The microscope was operated in the tapping mode for all samples at a frequency of 50 to 100 
$\mathrm{kHz}$. The images were processed and analysed using Nanoscope Analysis, version 1.80 .

\subsection{Transmission electron microscopy (TEM)}

The size and morphology of the particles in the dispersions were measured using a $120 \mathrm{kV}$ Biology TEM (Tecnai G2 spirit Biotwin, USA). Ten microliters of the solutions containing $0.02 \%(\mathrm{w} / \mathrm{w})$ NaCas were spread uniformly on a 200-mesh carbon coated copper grid and ten microliters of phosphotungstic acid were added onto the samples of the carbon coated copper grid to colour the samples. ${ }^{22}$ Subsequently, the excess liquid was removed using filter paper. ${ }^{23}$ Afterwards, the samples were put in a fume hood and dried at ambient temperature (25 $\left.{ }^{\circ} \mathrm{C}\right)$ overnight $(\sim 12 \mathrm{~h})$. Images were acquired at various magnifications under $120 \mathrm{kV}$.

\subsection{Scanning electron microscopy (SEM)}

Scanning electron micrographs of powdered samples containing chlorophylls and NaCas were obtained with an FEI Sirion 200 SEM instrument (FEI, USA). Briefly, fresh dispersion samples were lyophilized using a freeze dryer (Ningbo Xinzhi Biological Polytron Technologies, Inc., Ningbo, China) for $48 \mathrm{~h}$ and a small amount of powder was spread evenly on a $5 \times 5 \mathrm{~mm}$ piece of silicon wafer, the wafer was then adhered to a conductive carbon tape and sputter coated with a gold layer $(<10 \mathrm{~nm})$ using a magnetron sputtering device (HITACHI-E1045, Japan). Samples were then observed at $5 \mathrm{kV}$ utilizing an operating Zeiss Auriga microscope.

\subsection{Data analysis}

All the experiments were carried out in triplicate. The standard error and mean of the data were calculated for all the data processed. Analysis of variance (ANOVA) followed by the least significant difference (LSD) test was carried out to determine

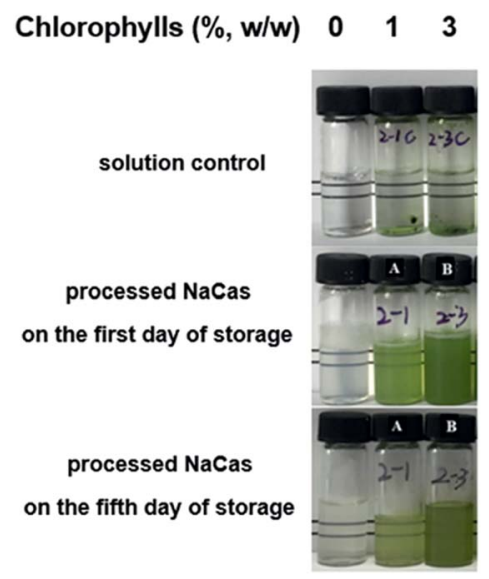

Fig. 1 The appearance of the control samples and $\mathrm{NaCas}$ dispersions with different concentrations of chlorophylls. The samples were stored in the dark at $30{ }^{\circ} \mathrm{C}$. Numbers $(2-1,2-3)$ on the body of bottles represent their batch. Solution control: freeze-dried chlorophylls redissolved in deionized water; processed $\mathrm{NaCas}$ on the first (fifth) day: $\mathrm{NaCas}$ with or without chlorophylls on the first (fifth) day of storage. any significant difference with $p$ set at 0.05 between treatments using SPSS version 16.0 software (SPSS Inc, IL, USA).

\section{Results and discussion}

\subsection{Storage stability of the dispersions}

The appearance, on the first and fifth day of storage, of the NaCas dispersions with 1 and $3 \%(\mathrm{w} / \mathrm{w})$ chlorophylls are presented in Fig. 1. All the dispersions had a uniform green appearance and the nanoparticles were dispersed in the aqueous systems in a homogeneous manner except for $\mathrm{H}$ NaCas. Phase separation was observed for H-NaCas on the third day which could be a result of overloading of the chlorophylls in H-NaCas.

The storage stability of the dispersions was evaluated by measuring the retention rate, hydrodynamic diameter $\left(D_{\mathrm{h}}\right)$, and zeta-potential of the chlorophylls (Fig. 2). The retention rate of
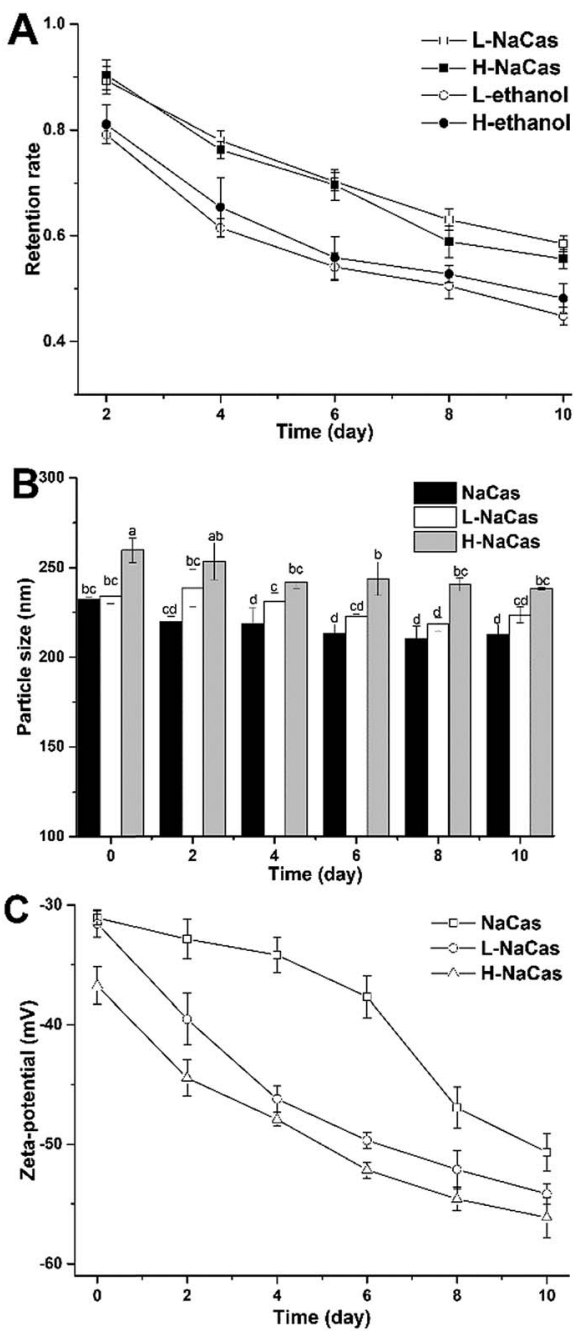

Fig. 2 Retention rate (A), particle size (B), and zeta-potential (C) of chlorophylls and dispersions during storage. The different letters above the bars indicate significant differences $(p<0.05)$. L-NaCas: $\mathrm{NaCas}$ with $1 \%(\mathrm{w} / \mathrm{w})$ chlorophylls; $\mathrm{H}-\mathrm{NaCas}$ : NaCas with $3 \%(\mathrm{w} / \mathrm{w})$ chlorophylls; L-ethanol: $1 \%(\mathrm{w} / \mathrm{W})$ chlorophylls in ethanol; $\mathrm{H}$-ethanol: $3 \%(\mathrm{w} / \mathrm{w})$ chlorophylls in ethanol. 

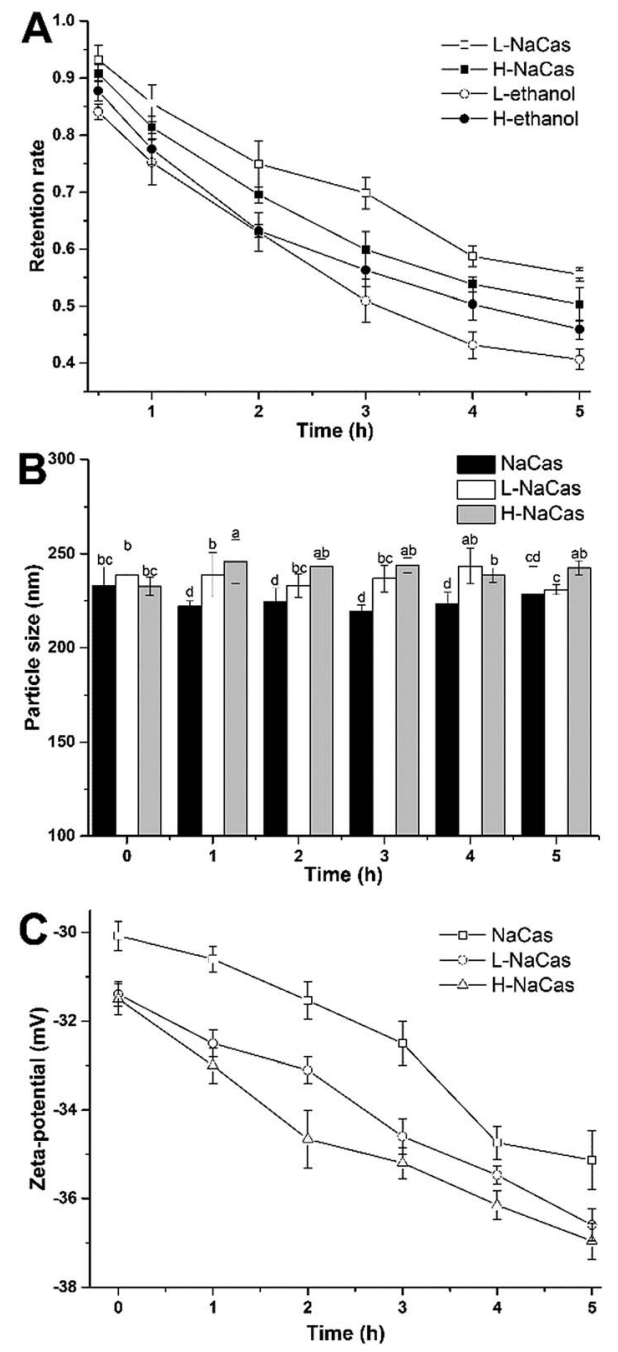

Fig. 3 Retention rate (A), particle size (B), and zeta-potential (C) of chlorophylls and dispersions after light exposure. Samples were placed $40 \mathrm{~cm}$ away from the lamp (9000 lx). Mean values in a row with different letters are significantly different $(p<0.05)$. L-NaCas: NaCas with $1 \%(\mathrm{w} / \mathrm{w})$ chlorophylls; $\mathrm{H}-\mathrm{NaCas}$ : NaCas with $3 \%(\mathrm{w} / \mathrm{w})$ chlorophylls; L-ethanol: $1 \%(\mathrm{w} / \mathrm{w})$ chlorophylls in ethanol; $\mathrm{H}$-ethanol: $3 \%(\mathrm{w} /$ w) chlorophylls in ethanol.

the chlorophylls in the dispersions was significantly higher than that of the chlorophylls in ethanol $(p<0.05)$ in which the chlorophylls retention rates for L-NaCas and $\mathrm{H}$-NaCas were $60.49 \pm 0.81 \%$ and $57.62 \pm 0.98 \%$, respectively, and the corresponding rates for the control were $44.81 \pm 0.79$ and $48.17 \pm$ $1.03 \%$ ( Fig. 2A). A similar phenomenon has been reported for NaCas which has been shown to decrease the degradation of some liposoluble pigments. ${ }^{24,25} D_{\mathrm{h}}$ of NaCas was $\sim 220 \mathrm{~nm}$ in accordance with the previous literature. ${ }^{26}$ The mean $D_{\mathrm{h}}$ were in the range of 221-245 $\mathrm{nm}$. $D_{\mathrm{h}}$ increased significantly with the chlorophylls content, which may be attributed to the higher concentration of chlorophylls $(p<0.05)$, and eventually led to a larger particle diameter. ${ }^{27}$ However, the mean particle size for each treatment decreased slightly with the storage time. As shown in Fig. 2C, the range of the zeta-potential value of NaCas, L-NaCas, and H-NaCas was -31.1 to $-50.7 \mathrm{mV},-31.7$ to
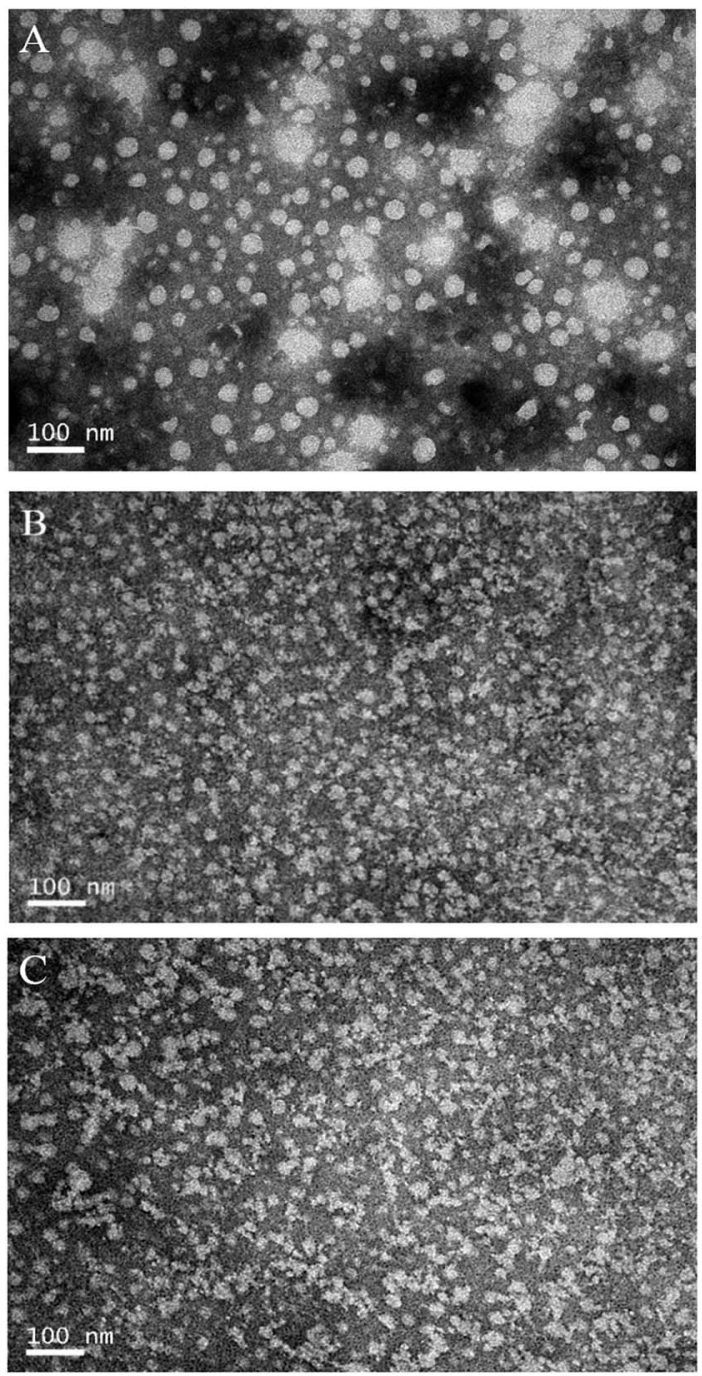

Fig. 4 TEM images of $\mathrm{NaCas}(\mathrm{A}), \mathrm{L}-\mathrm{NaCas}(\mathrm{B})$, and $\mathrm{H}-\mathrm{NaCas}(\mathrm{C})$. The images were taken at $12000 \times$ magnification (scale bar $=100 \mathrm{~nm}$ ). LNaCas: NaCas with $1 \%(w / w)$ chlorophylls; $\mathrm{H}-\mathrm{NaCas}$ : NaCas with $3 \%$ (w/w) chlorophylls.

$-52.2 \mathrm{mV}$, and -36.7 to $-56.2 \mathrm{mV}$, respectively, as the storage time increased, suggesting that the electrostatic repulsion in the dispersions became more effective at preventing particles from flocculating and coming together. ${ }^{12}$

\subsection{Photostability of the dispersions}

Chlorophylls have a brilliant color and many bioactivities. However, the instability of chlorophylls, caused by light, oxygen, heat, acid, and enzymes, especially the effect of light, mean that they are not favored for application as natural colorants in foods. ${ }^{7}$ The photostability of chlorophylls was evaluated by measuring their retention rate, particle size, and zeta-potential, as shown in Fig. 3. The retention rate of the chlorophylls was shown to decrease nonlinearly with the continuous visible light (Fig. 3A).

The retention rates of chlorophylls for L-NaCas and H-NaCas were $58.72 \pm 0.43 \%$ and $53.84 \pm 1.36 \%$, respectively, and the 

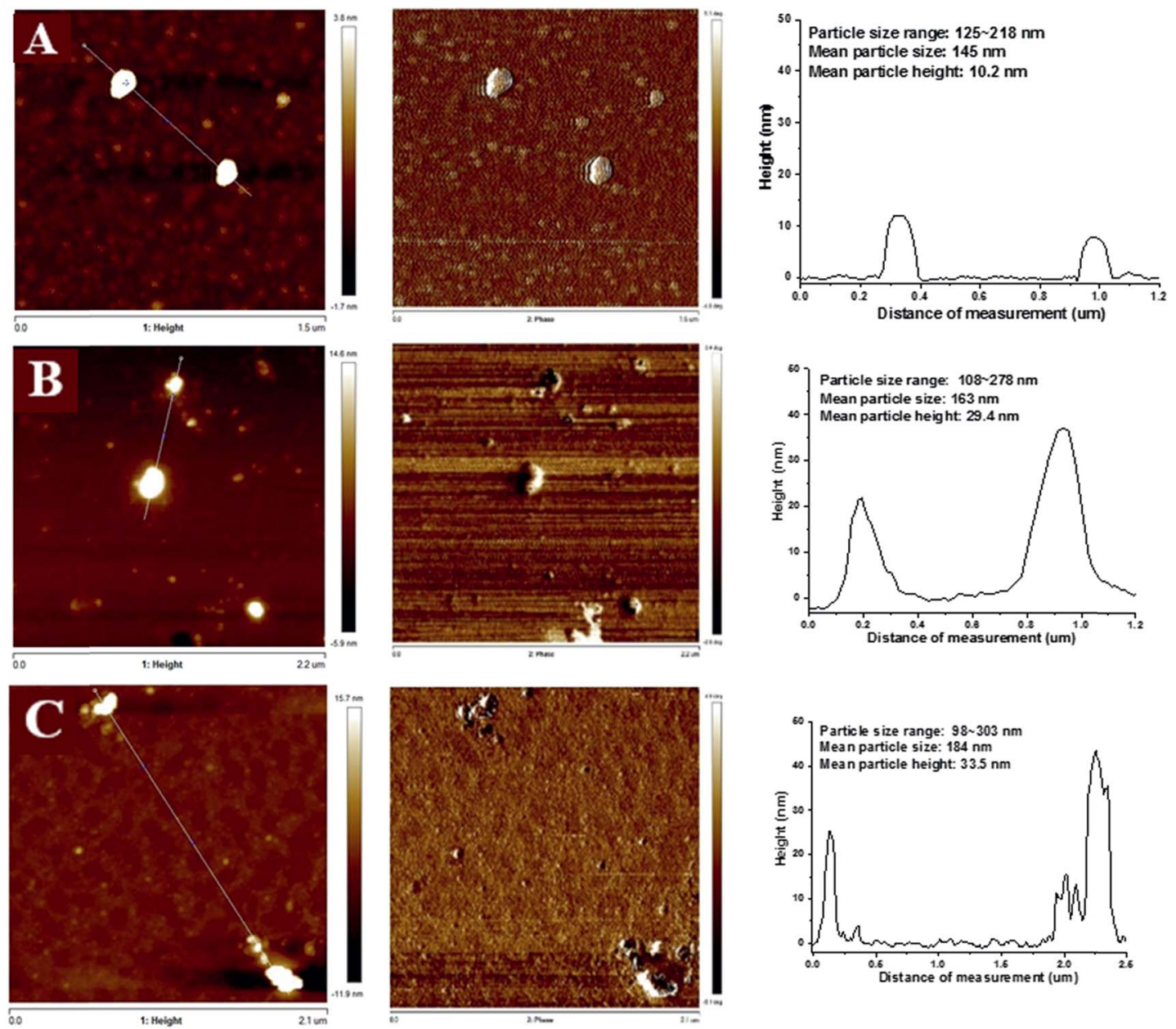

Fig. 5 AFM topographic images and phase diagrams of $\mathrm{NaCas}(\mathrm{A}), \mathrm{L}-\mathrm{NaCas}(\mathrm{B})$, and $\mathrm{H}-\mathrm{NaCas}$ (C). Plots next to images show the height of particles along the dashed line. L-NaCas: NaCas with $1 \%$ (w/w) chlorophylls; H-NaCas: NaCas with 3\% (w/w) chlorophylls.

corresponding rates for the control were $41.29 \pm 0.78 \%$ and $45.93 \pm 0.82 \%$, after $5 \mathrm{~h}$ of light treatment. The chlorophylls retention rate for L-NaCas was higher than that for H-NaCas due to the higher concentration of chlorophylls, as discussed previously. During $5 \mathrm{~h}$ radiation, no significant changes were found in $D_{\mathrm{h}}$, and the zeta-potential dimension increased with the light time. The results show that $\mathrm{L}$-NaCas treatment gives the highest retention rate of chlorophylls indicating that NaCas protected the chlorophylls.

\subsection{Morphology properties of NaCas-chlorophylls dispersions}

The morphological properties of NaCas, L-NaCas, and H-NaCas (fresh samples and powdered samples) were analyzed by TEM, AFM, and SEM. TEM was used to record the morphology of the nanoparticles and dispersions. AFM and SEM were utilized to characterize the topographic images and the mean particle height, and surface morphology of the powdered samples, respectively.

3.3.1 TEM analysis. The TEM images show the interior morphology of the nanoparticles and dispersions. TEM images of freshly prepared NaCas with or without chlorophylls are presented in Fig. 4. Particles of dispersions without chlorophylls were spherical and regular, which is in accordance with the previously published findings that all NaCas have a spherical structure. ${ }^{28}$ Larger sized particles were observed with increasing concentration of the chlorophylls, which was consistent with the trend observed in Fig. $2 \mathrm{~B}$ for $D_{\mathrm{h}}$. Additionally, the particles were more discrete and less spherical as the content of the chlorophylls increased, this may be because of the dissociation and assembly of NaCas. ${ }^{29}$ The absence of aggregation suggested that these particles could be dispersed well in the aqueous phase.

3.3.2 AFM analysis. The nanoscale structures of the dispersions were studied at pH 6.8 using AFM (Fig. 5), to analyze their mean particle size and height. The distribution of the particle size in different treatments (Fig. 5A-C) qualitatively agreed with the light scattering data shown in Fig. 2. Although the mean particle height followed the same trend with particle size. The particle height was much smaller than the particle size with the mean particle height of NaCas, L-NaCas, and H-NaCas being 10.2, 29.4, and $33.5 \mathrm{~nm}$, respectively (Fig. 5). The particle distribution of NaCas, which is mostly spherical in shape, was relatively narrow, 

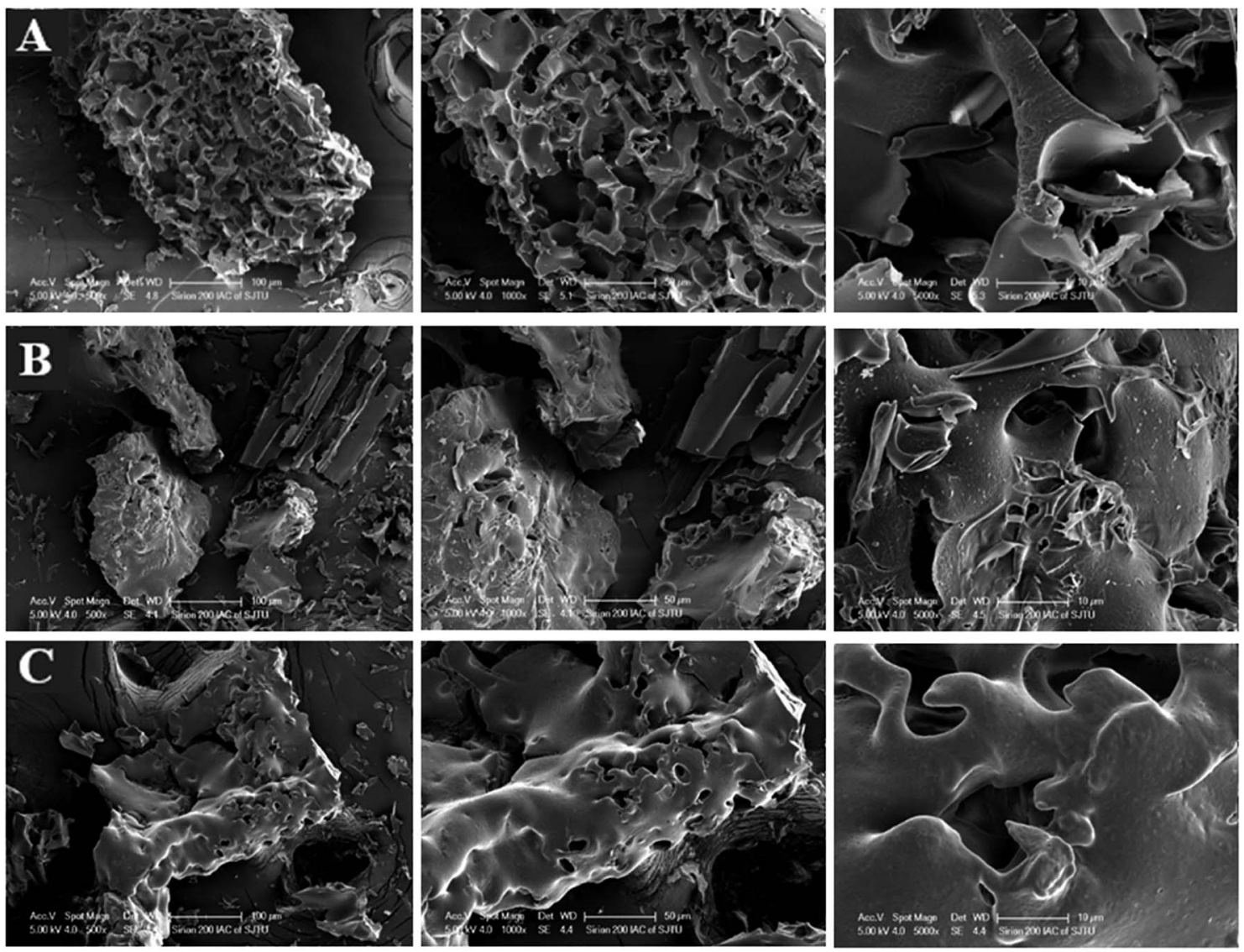

Fig. 6 SEM images of $\mathrm{NaCas}(\mathrm{A}), \mathrm{L}-\mathrm{NaCas}(\mathrm{B})$, and H-NaCas (C) under magnifications of 500, 1000, and $5000 \times$. L-NaCas: NaCas with 1\% (w/w) chlorophylls; H-NaCas: NaCas with $3 \%(w / w)$ chlorophylls.

125 to $218 \mathrm{~nm}$. In comparison, the nanoparticles in L-NaCas and $\mathrm{H}-\mathrm{NaCas}$, which are less regular in shape, had a wider particle size distribution from 107 to $278 \mathrm{~nm}$ and from 98 to $303 \mathrm{~nm}$, respectively. The assembly ability of caseins may be the cause of the discrepancies in the AFM images. ${ }^{30}$ A similar phenomenon was found for the morphology of nanoparticles containing liposoluble compound, these were less spherical and the particles had a wider particle size distribution. ${ }^{\mathbf{1 2 , 2 9}}$ The mean particle size of NaCas, LNaCas, and H-NaCas were 145, 163, and $184 \mathrm{~nm}$, respectively, indicating that the particle diameter increased with an increase in the chlorophylls concentration, this can be attributed to the deficiency in NaCas. ${ }^{12}$

3.3.3 SEM analysis. The morphology of lyophilized powder of NaCas with different concentrations of chlorophylls was investigated by SEM (Fig. 6). The morphology of NaCas presented as porous honeycomb structures (Fig. 6A) as a result of the porous network of spherical casein particle. ${ }^{31}$ The outer surface was much smoother and was less porous with an increasing chlorophylls concentration on the width of $50 \mu \mathrm{m}$ due to assembly of NaCas and chlorophylls (Fig. 6B and C). ${ }^{32}$ This phenomenon has also been reported by others that outer surface of NaCas was smooth after loading liposoluble compound. ${ }^{24}$ Dispersions of NaCas with or without chlorophylls showed considerable difference on morphology based on TEM, SEM, and AFM results. Chlorophylls might be the component causing the difference in the morphology since the same amount of ethanol was treated to the dispersions, which indicated that the addition of chlorophylls promoted NaCas dissociated and self-assembly to cause differences in morphology. Additionally, the $\mathrm{Mg}^{2+}$ in chlorophylls was also an important element for morphology differences, which was reported that it can increase the solution ionic strength to affect dissociation and re-association of NaCas. ${ }^{31}$

The change in morphology after adding chlorophylls was possibly caused by the interaction between the chlorophylls and NaCas. The interaction between NaCas and the chlorophylls was further confirmed through fluorescence spectroscopy.

\subsection{Fluorescence quenching of NaCas by chlorophylls: molecular interaction perspective}

Fluorescence spectroscopy was used to study the interactions between chlorophylls and caseins. Fluorescence is typically observed when the fluorophore of a molecule is fully or partially blocked. ${ }^{7}$ NaCas-chlorophylls showed an intense emission band concentrated around $341.6 \mathrm{~nm}$ as a result of fluorescence emission of tryptophan (Trp) and tyrosine (Tyr) residues upon excitation at $280 \mathrm{~nm} .{ }^{33}$ The intensity of the fluorescence emission of caseins at $341.6 \mathrm{~nm}$ gradually decreased with the increase in chlorophylls concentration (Fig. 7A-C), suggesting an interaction between the 

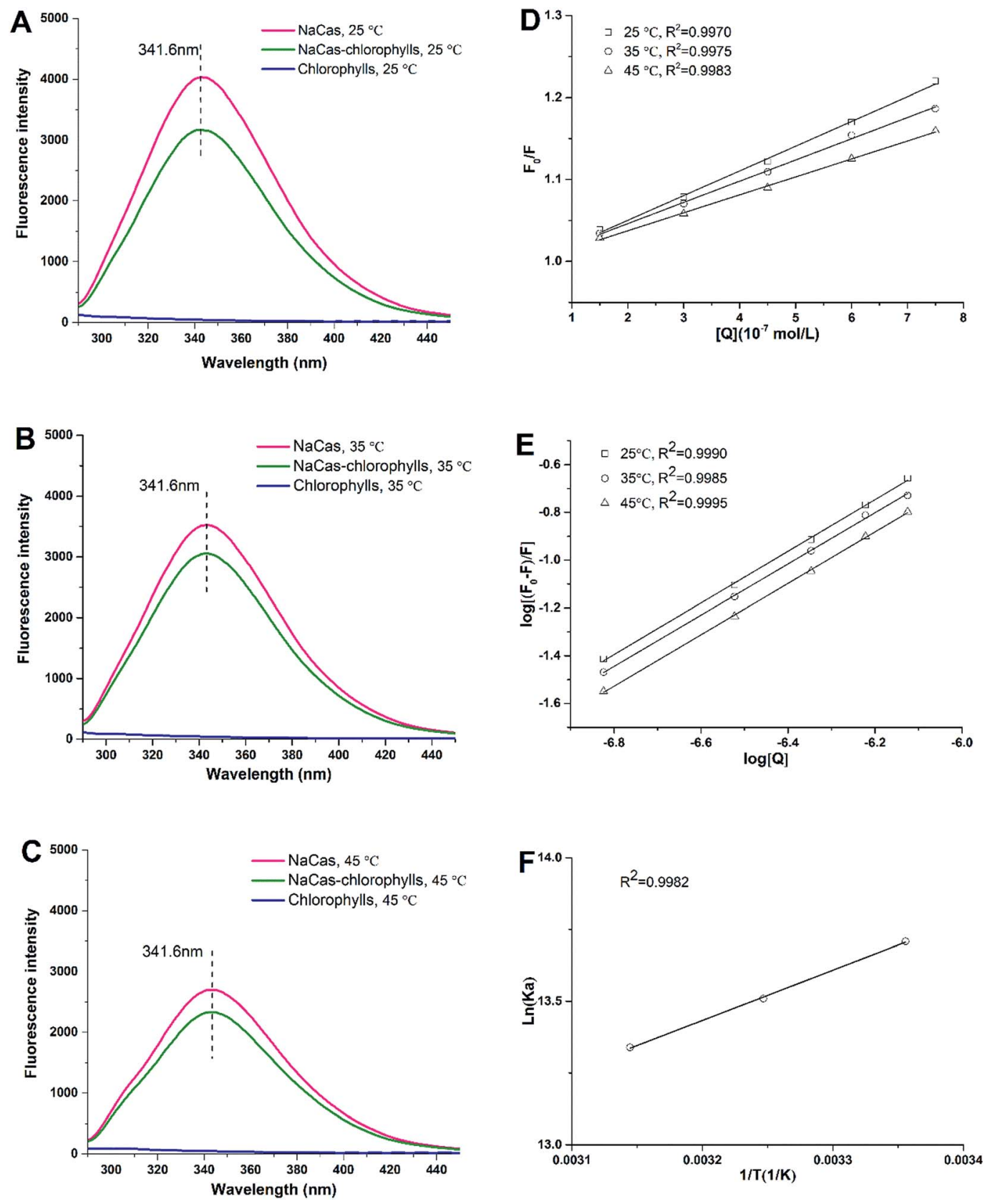

Fig. 7 Fluorescence intensity of $\mathrm{NaCas}$ with $1 \%$ or $3 \%(\mathrm{w} / \mathrm{w})$ chlorophylls in water at temperatures of $25^{\circ} \mathrm{C}(\mathrm{A}), 35^{\circ} \mathrm{C}(\mathrm{B})$, and $45^{\circ} \mathrm{C}(\mathrm{C})$. The SternVolmer plots (D) and the double-logarithm plot (E) show the quenching effect of chlorophylls on NaCas at 25,35 , and $45^{\circ} \mathrm{C}$. The Van't Hoff plot (F) of $\ln K$ as a function of $1 / T\left({ }^{\circ} \mathrm{C}\right)$ was calculated to reveal the interaction of NaCas and the chlorophylls molecules.

NaCas molecules and the chromophore groups of the chlorophylls. Chlorophylls binding to NaCas can be classified as dynamic or static quenching and may involve diffusion and collision

Table 1 Stern-Volmer quenching constants $\left(K_{\text {sv }}\right)$ and molecular quenching constants $\left(K_{\mathrm{q}}\right)$ for the interaction of chlorophyll with $\mathrm{NaCas}$ at 25,35 , and $45^{\circ} \mathrm{C}$

\begin{tabular}{llll}
\hline$T\left({ }^{\circ} \mathrm{C}\right)$ & $K_{\mathrm{sv}}\left(10^{5} \mathrm{M}^{-1}\right)$ & $K_{\mathrm{q}}\left(10^{13} \mathrm{M}^{-1} \mathrm{~s}^{-1}\right)$ & $R^{2}$ \\
\hline 25 & 2.26 & 2.26 & 0.9970 \\
35 & 2.03 & 2.03 & 0.9975 \\
45 & 1.64 & 1.64 & 0.9983
\end{tabular}

encounters. ${ }^{34}$ The quenching data were analyzed according to the Stern-Volmer equation: ${ }^{35}$

$$
F_{0} / F=1+K_{\mathrm{q}} \tau^{0}[\mathrm{Q}]
$$

where $F$ and $F_{0}$ are the fluorescence intensities with or without a quencher, respectively. $K_{\mathrm{q}}$ is the quenching rate constant of the biological macromolecule, [Q] is the concentration of the quencher, and $\tau^{0}\left(10^{-8} \mathrm{~s}\right)$ is the average lifetime of the fluorophore in the absence of the quencher.

The quenching parameters calculated from the modified Stern-Volmer plots are recorded in Table 1 . The good linearity 
Table 2 The binding constant $\left(K_{\mathrm{a}}\right)$ and number of binding sites of chlorophylls with the fluorophore of $\mathrm{NaCas}$ at temperature of 25,35 , and $45^{\circ} \mathrm{C}$. The enthalpy $(\Delta H)$, entropy $(\Delta S)$, and free energy $(\Delta G)$ change based on the van't Hoff equation

\begin{tabular}{lllllll}
\hline$T(\mathrm{~K})$ & $K_{\mathrm{a}}\left(10^{5} \mathrm{M}^{-1}\right)$ & $n$ & $R^{2}$ & $\Delta G\left(\mathrm{~kJ} \mathrm{~mol}^{-1}\right)$ & $\Delta H\left(\mathrm{~kJ} \mathrm{~mol}^{-1}\right)$ & $\Delta S\left(\mathrm{~J} \mathrm{~mol} \mathrm{~K}^{-1}\right)$ \\
\hline 298 & 8.99 & 1.0807 & 0.9990 & -14.75 & -6.31 \\
308 & 7.04 & 1.0722 & 0.9985 & -14.97 & -0.03 \\
318 & 6.21 & 1.0766 & 0.9995 & -15.32 &
\end{tabular}

of the Stern-Volmer plots, with all coefficients more than 0.9970 , suggests that a single quenching mechanism existed in the binding (Fig. 7D). The value of $K_{\mathrm{sv}}$ decreased with the rising temperatures, which indicates a reduced affinity of the chlorophylls with NaCas at higher temperatures. The $K_{\mathrm{q}}$ values were far higher than the maximal dynamic quenching constant $\left(2.0 \times 10^{10} \mathrm{M}^{-1} \mathrm{~s}^{-1}\right)$, which indicates that the interaction between NaCas and the chlorophylls was dominated by a static quenching mechanism. For the static quenching, the binding constant $\left(K_{\mathrm{a}}\right)$ of the fluorophore with the quencher is given by the following equation: ${ }^{36}$

$$
\log \left(\left(F_{0}-F\right) / F\right)=\log K_{\mathrm{a}}+n \log [\mathrm{Q}]
$$

where $n$ is the number of binding sites. The value of the apparent binding constant $K_{\mathrm{a}}$ can be obtained from the intercept of the plot of $\log \left[\left(F_{0}-F\right) / F\right]$ versus $\log [\mathrm{Q}]$ in Fig. 7E whereas the binding sites $n$ was the slope from eqn (5). The binding sites $n$ and binding constant $K_{\mathrm{a}}$ are presented in Table 2 . The value of $K_{\mathrm{a}}$ decreased with a rise in the temperature, as can be seen from the data presented in Table 2. The value of the binding sites $n$ was $\sim 1$, which signifies that only a single binding site of the chlorophylls was involved in the binding process. Moreover, the correlation coefficients of 0.9990 , 0.9985 and 0.9995 which were obtained with the increasing temperatures, confirmed that the interaction between NaCas and the chlorophylls were in accordance with the site-binding model outlined in eqn (5).

Four major noncovalent interaction forces, including hydrophobic and electrostatic interactions, hydrogen bonding, and van der Waals forces, are considered to exist between proteins and small molecules. Thermodynamic parameters $(\Delta H, \Delta G$, and $\Delta S)$ for protein reactions are the main factors contributing to protein stability. Noncovalent interaction forces and magnitudes of thermodynamic parameters can be modelled by the Van't Hoff plot: ${ }^{37}$

$$
\begin{gathered}
\ln K_{\mathrm{a}}=-\Delta H /(R T)+\Delta S / R \\
\Delta G=\Delta H-T \Delta S
\end{gathered}
$$

where $R$ is the gas constant $8.314 \mathrm{~J} \mathrm{~mol}^{-1} \mathrm{~K}^{-1}, T(\mathrm{~K})$ is the experimental temperature. $\Delta H, \Delta G$ and $\Delta S$ represent the enthalpy change, free energy change, and the entropy change, respectively. $K_{\mathrm{a}}$ is the binding constant at a corresponding temperature. The primary interaction can be determined as Van der Waal forces and/or hydrogen bonds (if $\Delta H<0$ and $\Delta S<0$ ), hydrophobic forces (if $\Delta H>0$ and $\Delta S>0$ ), or electrostatic interactions (if $\Delta H<0$ and $\Delta S>0$ ). ${ }^{36}$

The negative $\Delta H, \Delta S$, and $\Delta G$ suggest that the main interaction forces were van der Waals forces and hydrogen bonds and the binding process of chlorophylls to NaCas was spontaneous and exothermic at all experimental temperatures. $^{38}$

\subsection{Proposed mechanism of interaction of NaCas and chlorophylls}

The hypothesized mechanism of the structural changes in the study is presented in Fig. 8. The morphology of NaCas without chlorophylls was still spherical and regular as was the morphology of native NaCas while the particles were more discrete and less spherical as the content of the chlorophylls increased. ${ }^{39}$ The particle diameter increased as thechlorophylls concentration increased, which is in accordance with the AFM observations. For H-NaCas, some chlorophylls are not entrapped in the NaCas dispersion, which is consistent with the storage test results (Fig. 1). Compared with the L-NaCas, additional chlorophylls in H-NaCas might attract free NaCas to the particle, resulting in increased particle size, as shown in Fig. 8. Similar results were observed by AFM. The dissociation of NaCas increased the availability of hydrophilic sites of caseins for contact with the chlorophylls during mixing, along with the fluorescence spectroscopy results this suggests that the chlorophylls were entrapped in the NaCas dispersion via hydrogen bonds and van der Waals forces.

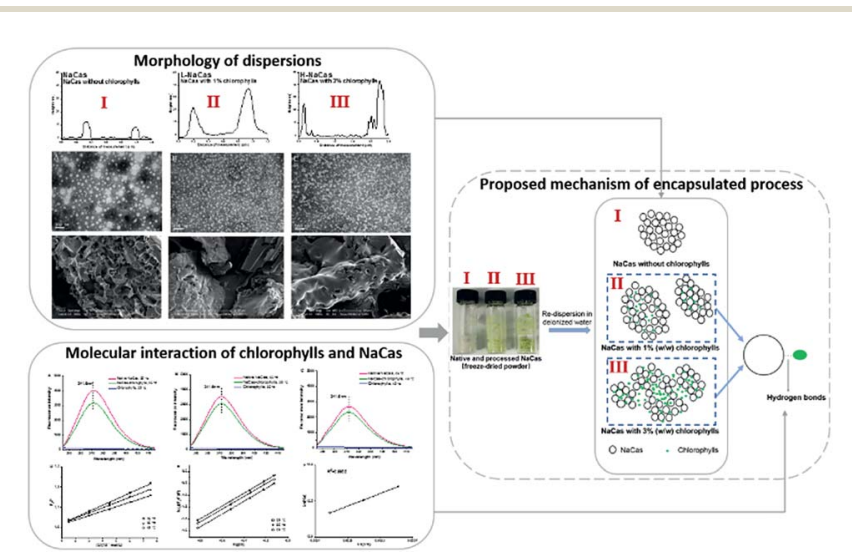

Fig. 8 Schematic mechanism of the structural changes of sodium caseinate (NaCas) and chlorophylls. 


\section{Conclusion}

In conclusion, chlorophylls dispersions containing sodium caseinate were prepared, this reduced the storage decomposition and light degradation of the chlorophylls in aqueous dispersion due to protection of the chlorophylls by NaCas. The self-assembly of NaCas with chlorophylls nanoparticles via van der Waals forces and hydrogen bonds by a spontaneous exothermic model was demonstrated by fluorescence quenching analyses. The topography observation using AFM further revealed that the size of the nanoparticles increase with an increase in the concentration of the chlorophylls, which is in line with the DLS determination. The steric hindrance and repulsive electrostatic interactions provided by hydrophilic NaCas prevents aggregation of colloidal particles of NaCas-chlorophylls. The data obtained from AFM and TEM, together with fluorescence spectroscopy results, suggests that the chlorophylls were entrapped in the NaCas dispersion via hydrogen bonds and van der Waals forces. The study showed that NaCas improved the stability of chlorophylls in the aqueous system and partially revealed the interaction of the chlorophylls and NaCas, this could potentially broaden the field of application of chlorophylls.

\section{Conflicts of interest}

There are no conflicts of interest to declare.

\section{Acknowledgements}

This study was funded by the Key R\&D Program of Guangdong Province (Grant No. 2018B020239001).

\section{References}

1 Y. R. Kang, J. Park, S. K. Jung and Y. H. Chang, Food Chem., 2017, 245, 943-950.

2 D. Marmion and U. B. Staff, Colorants for Foods, John Wiley \& Sons, Inc., 2007.

3 N. Koca, F. Karadeniz and H. S. Burdurlu, Food Chem., 2007, 100, 609-615.

4 G. Özkan and S. E. Bilek, Food Chem., 2015, 176, 152-157.

5 Y. R. Kang, J. Park, S. K. Jung and Y. H. Chang, Food Chem., 2018, 245, 943-950.

6 A. Subramoniam, V. V. Asha, S. A. Nair, S. P. Sasidharan, P. K. Sureshkumar, K. N. Rajendran, D. Karunagaran and K. Ramalingam, Inflammation, 2012, 35, 959-966.

7 Y. R. Kang, Y. K. Lee, Y. J. Kim and Y. H. Chang, Food Chem., 2019, 272, 337-346.

8 Chinese Standards for Food Additives, 2014, vol. GB27602014.

9 T. Tumolo and U. M. Lanfermarquez, Food Res. Int., 2012, 46, 451-459.

10 E. Lee, H. Ahn and E. Choe, Food Sci. Biotechnol., 2014, 23, 1061-1065.

11 D. Bednarczyk and D. Noy, JoVE, 2016, 109, 53410.
12 L. Wang and Y. Zhang, J. Agric. Food Chem., 2017, 65, 29902998.

13 D. S. Horne, Curr. Opin. Colloid Interface Sci., 2006, 11, 148153.

14 K. Pan, H. Chen, P. M. Davidson and Q. Zhong, J. Agric. Food Chem., 2014, 62, 1649-1657.

15 M. C. Ai, K. W. Tan, C. P. Tan and K. L. Nyam, Ind. Crops Prod., 2016, 80, 77-85.

16 H. Chen and Q. Zhong, Food Hydrocolloids, 2014, 35, 358366.

17 R. Wang, H. Wang, Y. Yao and Y. Chai, RSC Adv., 2018, 8, 36879-36885.

18 A. Maadane, N. Merghoub, T. Ainane, H. El Arroussi, R. Benhima, S. Amzazi, Y. Bakri and I. Wahby, J. Biotechnol., 2015, 215, 13-19.

19 J. Cheng, J. H. Liu, G. Prasanna and P. Jing, Int. J. Biol. Macromol., 2017, 105, 965-972.

20 S. H. Ching, B. Bhandari, R. Webb and N. Bansal, Food Hydrocolloids, 2015, 43, 165-171.

21 Y. Guan, J. Wu and Q. Zhong, Food Chem., 2016, 194, 787796.

22 K. Kalimuthu, B. C. Lubin, A. Bazylevich, G. Gellerman, O. Shpilberg, G. Luboshits and M. A. Firer, J. Nanobiotechnol., 2018, 16, 34.

23 Y. Jiao, D. Li, C. Liu, Y. Chang, J. Song and Y. Xiao, RSC Adv., 2018, 8, 31372-31381.

24 Y. Zhang and Q. Zhong, Food Hydrocolloids, 2013, 33, 1-9.

25 F. P. Chen, B. S. Li and C. H. Tang, J. Agric. Food Chem., 2015, 63, 3559-3569.

26 T. C. P. Moreira, R. N. Pereira, A. A. Vicente and R. L. da Cunha, Food Res. Int., 2018, 116, 628-636.

27 K. Hu, X. Huang, Y. Gao, X. Huang, H. Xiao and D. J. McClements, Food Chem., 2015, 182, 275-281.

28 L. Wang and Y. Zhang, J. Agric. Food Chem., 2017, 65, 29902998.

29 K. Pan, Q. Zhong and S. J. Baek, J. Agric. Food Chem., 2013, 61, 6036-6043.

30 N. N. Silva, A. Bahri, F. Guyomarc'h, E. Beaucher and F. Gaucheron, Dairy Sci. Technol., 2015, 95, 75-86.

31 A. Bahri, M. Martin, C. Gergely, S. Marchesseau and D. Chevalier-Lucia, Food Hydrocolloids, 2018, 83, 53-60.

32 M. A. D. L. Fuente, Trends Food Sci. Technol., 1998, 9, 281-288. 33 A. Sahu, N. Kasoju and U. Bora, Biomacromolecules, 2008, 9, 2905-2912.

34 L. K. Fraiji, D. M. Hayes and T. Werner, J. Chem. Educ., 1992, 69, 424.

35 M. K. Jain, J. Biochem. Biophys. Methods, 1984, 9, 181.

36 Y. Shu, W. Xue, X. Xu, Z. Jia, X. Yao, S. Liu and L. Liu, Food Chem., 2015, 173, 31-37.

37 M. Barathi, A. S. K. Kumar, C. U. Kumar and N. Rajesh, RSC $A d v .$, 2014, 4, 53711-53721.

38 P. D. Ross and S. Subramanian, Biochemistry, 1981, 20, 30963102.

39 L. Wang and Y. Zhang, J. Agric. Food Chem., 2017, 65, 29902998. 